\title{
Níveis dos sintomas climatéricos em mulheres fisicamente ativas e insuficientemente ativas
}

\author{
Levels of climacteric symptoms in physically active and insufficiently \\ active women \\ Ana Marina Pacheco Probo', Nathanael Ibsen da Silva Soares ${ }^{1}$, Vernon Furtado da Silva², Patrícia Uchôa Leitão Cabral
}

\begin{abstract}
Resumo
O objetivo do estudo foi avaliar o nível de sintomatologia em mulheres fisicamente ativas e insuficientemente ativas. A amostra foi composta por 60 mulheres entre 40 a 60 anos (média 49,4), sendo 30 fisicamente ativas e 30 insuficientemente ativas. Características sociodemográficas e clínicas foram avaliadas por instrumentos padrão, a sintomatologia climatérica pelo Índice Menopausal de Blatt-Kupperman (IMBK) e o nível de atividade física, pela versão curta do International Physical Activity Questionnaire (IPAQ). O programa SSPS, versão 18.0, foi utilizado em abrangências descritivas e inferenciais. O Test $t$ student e o Test $\chi^{2}$ revelaram diferenças não significativas em características sociodemográficas entre os grupos ( $p>0.05$ ). Dados do IMBK e os referentes a todos os sintomas, com exceção da depressão e zumbidos, revelaram níveis significativos, sendo todos mais baixos para as mulheres ativas $(\mathrm{p}<0.05)$. Uma Análise de regressão linear relativa aos sintomas climatéricos mostrou que a prática regular de atividade física, pode reduzir o valor do IMBK até 12 pontos. A atividade física regular faz diminuir a intensidade dos sintomas climatéricos em mulheres de meia idade.
\end{abstract}

\section{Palavras-chave}

Atividade motora; Menopausa; Exercício.

\begin{abstract}
The study purpose was evaluate the level of climacteric symptoms in physically active and insufficiently actives women. Sample consisted of 60 women aged 40 to 60 years (mean 49.4), being 30 physically active and 30 sedentary, being all of them residents in Teresina - PI. Sociodemographic and clinical characteristics were assessed by standard instruments, the climacteric symptoms by the Menopausal Index of Blatt-Kupperman (MIBK) and the level of physical activity, by the short version of the International Physical Activity Questionnaire (IPAQ). The SSPS program, version 18.0, was used in descriptive and inferential scopes. The Student $t$ and the $\chi^{2}$ tests revealed no significant differences in demographic characteristics between the groups ( $p>0.05)$. Data from the MIBK and from all of the symptoms itens, exception for depression and tinnitus, revealed significant differences between groups, being the lower levels related with the active women $(p<0.05)$. A linear regression analysis on the climacteric symptoms showed that regular physical activity may decrease the value of BMI in 12 points. Regular physical activity can decreases the intensity of climateric symptoms in middle-aged women.
\end{abstract}

\section{Keywords}

Motor Activity; Menopause; Exercise.

\section{Introdução}

Nas últimas décadas o interesse pelo estudo da qualidade de vida de mulheres no climatério tem aumentado devido em grande parte, ao aumento da expectativa de vida, fato que permite uma previsão de que a população feminina possa viver cerca de um terço de suas vidas após a menopausa ${ }^{1}$. Estudos recentes têm mostrado associações entre a idade de ocorrência da menopausa e

1 Universidade Estadual do Piauí. Departamento de Educação Física. Teresina, Piauí, Brasil. 2 Universidade do Estado do Rio de Janeiro. Instituto de Educação Física e Desportos. Rio de Janeiro, Brasil. aumento da taxa de mortalidade, o que representa problema de saúde pública e um importante desafio para a criação e efetivação de políticas públicas voltadas para atenção em saúde da mulher climatérica² ${ }^{2}$.

O climatério, condição que tem início por volta dos 40 anos e estende-se até os 65 anos de idade pode ser caracterizado como sendo um fenômeno fisiológico originado pelo esgotamento dos folículos ovarianos que leva a mulher a um estado de hipoestrogenismo progressivo, consumando-se com a interrupção definitiva dos ciclos menstruais (menopausa) e aparecimento de sintomas característicos ${ }^{3}$. 
As alterações biológicas e psicossociais que acometem a mulher no período do climatério têm impacto negativo sobre a sua saúde e bem-estar. Dentre as alterações biológicas observam-se sintomas típicos como: distúrbios menstruais, sudorese, fogachos, cefaleia, palpitação, fraqueza, tontura, irritabilidade, insônia, depressão, ansiedade, nervosismo, dificuldade de concentração e esquecimento ${ }^{4,5}$.

Em um longo prazo, o decréscimo na secreção dos hormônios sexuais femininos predispõe à mulher ao acometimento de doenças crônico-degenerativas (Exemplo: Osteoporose, doenças coronarianas, hipertensão e dislipidemias), doenças estas que além de interferirem no estado geral de saúde, tendem a influenciar, também, certos aspectos da sua sexualidade ${ }^{6}$.

As terapias hormonais repositoras têm avançado na busca por sanar, na medida do possível, os problemas associados ao decréscimo de hormônios que ocorrem em função do climatério. Nesta direção, a terapia baseada no uso de estrógenos passou recentemente a ser indicada com critérios mais rígidos, os quais buscam formas interativas do método com outras práticas paralelas, tais como adequação nutricional e aderência à prática de atividades físicas ${ }^{7}$.

Alguns estudos já apoiam a prática regular de atividades físicas para a redução da intensidade dos sintomas climatéricos ${ }^{8,9}$. Além disto, dados já consolidados na literatura têm mostrado que a prática regular de atividades físicas é uma intervenção essencial na prevenção e no tratamento de diversas patologias decorrentes do envelhecimento, entre elas a hipertensão, diabetes mellitus tipo 2, as dislipidemias e a aterosclerose ${ }^{10}$.

A prática de exercício físico contínuo influencia na secreção de $\beta$-endorfinas hipotalâmica, aliviando as ondas de calor e melhorando o humor durante o climatério ${ }^{11}$. Tairova e De Lorenzi ${ }^{12}$ mostrou que as mulheres fisicamente ativas de sua pesquisa relataram menor intensidade de sintomas do domínio psicológico em comparação com as mulheres sedentárias.

Em Portugal, Guimarães e Baptista ${ }^{13}$ realizaram um estudo com 104 mulheres de meia idade, agrupadas de acordo com a duração da sessão diária de atividade física que realizavam (grupo A - $30 \mathrm{~min} /$ dia; grupo B - 30-60 min/dia; grupo C - 60 $\mathrm{min} / \mathrm{dia}$ ). Ao final de 12 semanas de intervenção foi constatado que as mulheres com prática de atividade física de $60 \mathrm{~min} /$ dia mostraram diminuição da intensidade dos sintomas climatérios, bem como melhor percepção dos domínios psicológico e social da qualidade de vida. Estes dados sugerem que a adoção da prática regular de atividades físicas moderadas pode exercer efeitos positivos significativos na saúde da mulher climatérica.

Estudo recente mostrou que o maior nível de atividade física esteve associado com melhorias nos sintomas somáticos e físicos ${ }^{14}$. Em uma outra pesquisa as mulheres praticantes de exercícios físicos apresentaram menor intensidade em todos os sintomas climatéricos, em comparação com as que não praticavam exercícios físicos ${ }^{15}$.

Sob a perspectiva da literatura acima apresentada, a prática regular de exercícios físicos pode ser considerada uma opção terapêutica para o alívio dos sintomas da menopausa e melhora da qualidade de vida de mulheres de meia idade ${ }^{9}$. Embora assim, as comparações efetivadas na relação entre atividades físicas e climatério têm, quase sempre, focado à distância extremo nos níveis estudados. Ou seja, na relação entre praticantes e não praticantes de atividades físicas. Diante deste fato, a questão que emerge seria o da possibilidade de que um nível baixo de atividade física pudesse ser suficiente para remediar os efeitos do climatério. Esta questão, portanto, permeou o objetivo principal deste estudo, que buscou comparar o nível de sintomatologia climatérica entre mulheres fisicamente ativas e insuficientemente ativas. 


\section{Métodos}

Estudo descritivo com delineamento transversal que compreendeu uma amostra de mulheres saudáveis com idades entre 40 a 60 anos, recrutadas a partir de um parque público para prática de atividades físicas na cidade de Teresina - PI. Foi utilizado o processo de amostragem não probabilística por conveniência para seleção das participantes. Este critério de amostragem por conveniência deveu-se ao fato da dificuldade de se encontrar um número maior de participantes com as características próprias à essência do questionamento da pesquisa. Assim sendo, as inferências feitas, pós resultados, foram limitadas às características dos grupos das mulheres selecionadas.

Em observância aos critérios de exclusão, as mulheres com problemas neuropsiquiátricos ou distúrbios endócrinos, e aquelas que usavam medicamentos que interferem na qualidade de vida (por exemplo, terapia hormonal, antidepressivos, ansiolíticos ou antipsicóticos) não foram aceitas à participação no estudo. Também não foram aceitas aquelas que se classificaram nos padrões de inativas segundo o IPAQ. A consideração dos critérios de inclusão e exclusão fez resultar uma amostra final de 30 mulheres fisicamente ativas e 30 insuficientemente ativas, dentre as 96 que inicialmente atenderam a solicitação de participação.

Foram utilizados três instrumentos nesta pesquisa: Questionário contendo dados sociodemográficos, o Índice Menopausal de Blatt e Kupperman (IMBK) ${ }^{16} \mathrm{e}$ International Physical Activity Questionnaire (IPAQ) ${ }^{17}$.

Os questionários foram aplicados por um mesmo pesquisador, sendo entregue a cada participante uma cópia dos mesmos para que pudesse acompanhar as questões lidas em voz alta e as respostas assinaladas nos formulários pelo aplicador. O projeto foi aprovado pelo Comitê de Ética da Universidade Estadual do Piauí (CEP-UESPI), sob o n. 785.261. Todas as mulheres participantes da pesquisa foram devidamente esclarecidas e assinaram o Termo de Consentimento Livre e Esclarecido (TCLE).

O instrumento utilizado para avaliar os sintomas climatéricos foi o Índice Menopausal de Blatt e Kupperman (IMBK), que permite avaliar quantitativamente a ocorrência global de 11 sintomas ou queixas (sintomas vasomotores, insônia, parestesia, nervosismo, melancolia, vertigem, fraqueza, artralgia/mialgia, dor de cabeça, palpitações e zumbido). Para cada sintoma é estabelecido um peso diferente, de acordo com a intensidade e prevalência (ausente $=0$, leves $=1$, $\operatorname{moderados}=2$, intensos $=3$ ) e multiplicados por um escore específico para cada sintoma. O somatório dos valores de cada sintoma resulta em um índice menopausal, que deve ser classificado de acordo com a intensidade dos sintomas em: leves $(\leq 19)$, moderado $(20-35)$ ou grave $(>35)^{16}$.

$\mathrm{Na}$ avaliação do nível de atividade física, o instrumento utilizado foi o International Physical Activity Questionnaire (IPAQ) em sua versão curta. O instrumento avalia a frequência (em dias), e a duração (em minutos) de atividades de lazer, ocupacionais, de locomoção e de trabalhos domésticos, realizados por mais de dez minutos contínuos durante uma semana normal ${ }^{17}$. A pontuação foi obtida pela soma da quantidade de dias e minutos ou horas das atividades realizadas na semana anterior ao preenchimento do questionário. As participantes foram categorizadas em sedentárias (I), quando não realizaram atividade física por pelo menos 10 minutos contínuos durante a semana; insuficientemente ativas (IA), quando a atividade física na última semana ficou entre 10 e 149 minutos; e ativas (A), quando a duração da atividade física atingiu ou superou os 150 minutos semanais. Para os objetivos deste estudo, as mulheres selecionadas foram as que atenderam aos critérios de ativas e insuficientemente ativas.

A análise estatística foi realizada por meio do programa Statistical Package for the Social Science (SPSS), versão 18.0. Foi realizada a estatística descritiva em mé- 
dia, desvio padrão das variáveis quantitativas e frequência absoluta e percentual das variáveis qualitativas. Primeiramente, aplicou-se o teste de Kolmorogov-Smirnov para avaliar a normalidade das variáveis. Após a verificação de que o fator idade e as variáveis antropométricas seguiram padrão de normalidade, optou-se, em sequência, pela aplicação de testes paramétricos. Em virtude de o IMBK apresentar padrão de normalidade, decidiu-se, também, pela utilização de testes paramétricos.

Para verificar diferença entre as médias dos grupos aplicou-se o teste t student (dados paramétricos) e Mann-Whitney (dados não paramétricos). Para se verificar a possível associação entre as variáveis qualitativas aplicou-se o teste qui-quadrado $\left(\chi^{2}\right)$. Foi utilizado também a analise de regressão linear simples para prever o valor do IMBK baseado no nível de atividade física. Considerou-se significativo um valor de $\mathrm{p}<0,05$ para cada uma das comparações efetivadas.

\section{Resultados}

A média de idade das mulheres avaliadas foi de 49,4 anos, a maioria era de cor branca $(68,3 \%)$, casada $(66,7 \%)$, com renda familiar acima ou igual a quatro salários mínimos (60\%). Um quantitativo de $30 \%$ das mulheres possuía ensino superior completo, sendo que $15 \%$ delas tinham pós-graduação. Os dados dispostos na Tabela 1, no que tange as características sociodemográficas, mostram que quando investigados inferencialmente, não apresentaram diferenças significativas entre os grupos $(\mathrm{p}<0,05)$.

TABELA 1 - Características sociodemográficas da amostra da pesquisa segundo nível de atividade física.

\begin{tabular}{|c|c|c|c|c|c|c|c|}
\hline & \multicolumn{2}{|c|}{ Insuf. Ativas ( $\mathrm{n}=30$ ) } & \multicolumn{2}{|c|}{ Ativas $(n=30)$} & \multicolumn{2}{|c|}{ Total $(n=60)$} & \multirow[b]{2}{*}{$p$} \\
\hline & $\mathrm{N}$ & $\%$ & $\mathrm{~N}$ & $\%$ & $\mathrm{~N}$ & $\%$ & \\
\hline \multicolumn{8}{|l|}{ Faixa etária } \\
\hline 39-49 anos & 17 & 56,7 & 16 & 53,3 & 33 & 55,0 & 0,795 \\
\hline $50-60$ anos & 13 & 43,3 & 14 & 46,7 & 27 & 45,0 & \\
\hline Média (D.P) & \multicolumn{2}{|c|}{$49,1(5,0)$} & \multicolumn{2}{|c|}{$49,6(6,9)$} & \multicolumn{2}{|c|}{$49,4(6,0)$} & $0,735^{k}$ \\
\hline Cor & & & & & & & 0,052 \\
\hline Branco & 17 & 56,7 & 24 & 80,0 & 41 & 68,3 & \\
\hline Não branco & 13 & 43,3 & 06 & 20,0 & 19 & 31,7 & \\
\hline Estado civil & & & & & & & 0,348 \\
\hline Solteiro (a) & 0 & 0,0 & 03 & 10,0 & 03 & 5,0 & \\
\hline Casado(a)/União Estável & 21 & 70,0 & 19 & 63,3 & 40 & 66,7 & \\
\hline Separado (a) & 06 & 20,0 & 06 & 20,0 & 12 & 20,0 & \\
\hline Viúvo (a) & 03 & 10,0 & 02 & 6,7 & 05 & 8,3 & \\
\hline Escolaridade & & & & & & & 0,124 \\
\hline Fund. Incompleto & 03 & 10,0 & 0 & 0,0 & 03 & 5,0 & \\
\hline Fund. Completo & 01 & 3,3 & 0 & 0,0 & 01 & 1,7 & \\
\hline Médio Completo & 11 & 36,7 & 07 & 23,3 & 18 & 30,0 & \\
\hline Superior Incompleto & 06 & 20,0 & 05 & 16,7 & 11 & 18,3 & \\
\hline Superior Completo & 07 & 23,3 & 11 & 36,7 & 18 & 30,0 & \\
\hline Pós-Graduação & 02 & 6,7 & 07 & 23,3 & 09 & 15,0 & \\
\hline Renda Familiar & & & & & & & 0,408 \\
\hline $1-2 S M$ & 02 & 6,7 & 0 & 0,0 & 02 & 3,3 & \\
\hline $2-3 S M$ & 05 & 16,7 & 03 & 10,0 & 08 & 13,3 & \\
\hline $3-4$ SM & 06 & 20,0 & 08 & 26,7 & 14 & 23,3 & \\
\hline$\geq 4 S M$ & 17 & 56,7 & 19 & 63,3 & 36 & 60 & \\
\hline
\end{tabular}

SM: Salário Mínimo (R\$ 678,0). ${ }^{a}$ Teste Qui-quadrado $\left(c^{2}\right) ;{ }^{b}$ t student. 
Os resultados apontaram que mais de $76 \%$ das mulheres com nível educacional elevado atenderam as recomendações de atividade física. Contrariamente, $50 \%$ das mulheres de maior escolaridade não atenderam as recomendações de atividade física. No geral, o IPAQ revelou que $30(50 \%)$ das mulheres pesquisadas foram classificadas como insuficientemente ativa.

$\mathrm{Na}$ tabela 2 observa-se que os escores dos sintomas que envolvem o IMBK, com exceção da depressão, mostraram-se mais elevados no grupo de mulheres insuficientemente ativas quando comparados àquelas ativas. $\mathrm{O}$ índice geral do IMBK apresentou-se mais baixo nas mulheres ativas $(9,4)$, quando comparado às mulheres insuficientemente ativas $(21,4)$, indicando maior intensidade na sintomatologia climatérica neste grupo. A análise por meio do teste de Mann-Whitney revelou diferenças estatisticamente significativas $(p<0,05)$ em todos os sintomas (exceto depressão e presença de zumbidos).

TABELA 2 - Sintomas do climatério pelo Índice Menopausal de Blatt e Kupperman (IMBK) das participantes da pesquisa, de acordo com o nível de atividade física.

\begin{tabular}{lccccc}
\hline \multirow{2}{*}{ IMBK/Sintomas } & \multicolumn{2}{c}{ Insuf. Ativas $(\mathrm{n}=30)$} & \multicolumn{2}{c}{ Ativas $(\mathrm{n}=30)$} & \multirow{2}{*}{$\mathrm{p}^{\text {a }}$} \\
\cline { 2 - 5 } & Média & D.P & Média & D.P & \\
\hline Ondas de calor & 6,8 & 4,6 & 2,9 & 3,3 & 0,001 \\
\hline Parestesia & 0,9 & 1,7 & 0,2 & 0,6 & 0,045 \\
\hline Insônia & 2,9 & 2,0 & 1,5 & 1,6 & 0,008 \\
\hline Impaciência e Nervosismo & 3,5 & 1,9 & 1,9 & 2,0 & 0,001 \\
\hline Depressão & 0,3 & 0,6 & 0,3 & 0,7 & 0,584 \\
\hline Fadiga ou Cansaço & 2,1 & 0,8 & 0,9 & 0,7 & $<0,001$ \\
\hline Artralgia & 1,6 & 1,0 & 0,4 & 0,6 & $<0,001$ \\
\hline Cefaleia & 1,9 & 0,9 & 0,8 & 0,7 & $<0,001$ \\
\hline Palpitação & 1,2 & 1,8 & 0,5 & 1,3 & 0,043 \\
\hline Zumbidos & 0,2 & 0,5 & 0,1 & 0,4 & 0,410 \\
\hline Índice geral (IMBK) & 21,4 & 10,0 & 9,4 & 7,4 & $<0,001$ \\
\hline
\end{tabular}

Teste Mann-Whitney

A tabela 3 mostra a associação significativa entre a intensidade dos sintomas pelo IMBK e o nível de atividade física das mulheres $(\mathrm{p}<0,001)$, sendo possível verificar que $93,8 \%$ das mulheres ativas foram classificadas com intensidade leve dos sintomas. Nas mulheres insuficientemente ativas apenas 33,3\% destas tinham sintomas leves e $60 \%$ sintomas de intensidade moderada.

TABELA 3 - Classificação dos sintomas do climatério pelo Índice Menopausal de Blatt e Kupperman (IMBK), segundo nível de atividade física.

\begin{tabular}{lccccccc}
\hline \multirow{2}{*}{ Sintomas } & \multicolumn{2}{c}{ Insuf. Ativa $(\mathrm{n}=30)$} & \multicolumn{2}{c}{ Ativas $(\mathrm{n}=30)$} & \multicolumn{2}{c}{ Total $(\mathrm{n}=60)$} & \\
\cline { 2 - 6 } & $\mathrm{n}$ & $\%$ & $\mathrm{n}$ & $\%$ & $\mathrm{n}$ & $\%$ & $\mathrm{p}^{\mathrm{a}}$ \\
\hline Leve & 10 & 33,3 & 28 & 93,8 & 38 & 63,3 & \\
\hline Moderado & 18 & 60,0 & 01 & 3,3 & 19 & 31,7 & $<0,001$ \\
\hline Severo & 02 & 6,7 & 01 & 3,3 & 03 & 5,0 & \\
\hline
\end{tabular}

Teste Qui-quadrado $\left(c^{2}\right)$

A análise efetivada por uma regressão linear (Tabela 4) faz denotar que as mulheres do grupo ativas quando comparadas às mulheres do grupo insuficientemente ativas têm chances a reduzir, em 12 pontos, os escores no IMBK $(\mathrm{p}<0.05)$, 
mostrando uma tendência positiva dos efeitos da prática regular de atividades físicas na minimização dos sintomas climatéricos da mulher.

TABELA 4 - Regressão linear relativa aos sintomas do climatério de acordo com Índice Menopausal de Blatt e Kupperman (IMBK) da amostra segundo nível de atividade física.

\begin{tabular}{lcccc} 
& \multicolumn{3}{c}{ IMBK } & \\
\cline { 2 - 5 } & $\beta$ & $($ IC95\%) & $p$ & $r^{2}$ \\
\hline Constante & 21,4 & & $<0,001$ & 0,324 \\
\hline Insuf. Ativas & ref. & & & \\
\hline Ativas & $-12,0^{*}$ & $-16,0 ;-7,45$ & $<0,001$ & \\
\hline
\end{tabular}

$\beta$ : coeficiente de regressão linear; IC95\%: intervalo de confiança de 95\%; $r^{2}$ : coeficiente de determinação ajustado; IMBK $=b_{0}(21,4)+b_{1}(-12,0)$; *Variável ajustada para idade (anos).

\section{Discussão}

Os resultados do presente estudo sugerem que a prática de atividades físicas parece influenciar positivamente a diminuição dos sintomas climatéricos e sua intensidade. Neste estudo, foi encontrada diferença significativa $(\mathrm{p}<0,001)$ no IMBK entre o grupo de mulheres ativas e não suficientemente ativas, indicando menor incidência e intensidade de sintomas climatéricos nas mulheres ativas. Os sintomas analisados como: ondas de calor, parestesia, insônia, impaciência e nervosismo, fadiga ou cansaço, artralgia, cefaleia, palpitação e zumbidos mostraram médias de escores menos elevados e significativos $(\mathrm{p}<0,05)$ nas mulheres ativas, quando comparadas às insuficientemente ativas.

Nossos resultados somam-se positivamente aos de outros estudos que também avaliaram a relação entre a atividade física e a sintomatologia climatérica. Por exemplo, Canário et al. ${ }^{18}$ avaliou 370 mulheres climatéricas ativas e não ativas, e verificou que em todos os sintomas do IMBK, assim como em todos os domínios do Menopause Rating Scale (MRS), as ativas relataram menor intensidade e menor prevalência de sintomas climatéricos quando comparadas ao relato das insuficientemente ativas.

Em nosso estudo, foi possível evidenciar que os níveis de intensidade dos sintomas climatéricos foram significativamente diferentes entre os grupos avaliados. O melhor nível de sintoma pelo IBMK (sintomas leves) foi atribuído a 93\% das mulheres ativas, enquanto a maioria $(60 \%)$ das mulheres insuficientemente ativas apresentaram níveis de sintomas moderados. Este percentual foi considerado elevado entre as insuficientemente ativas, ao considerarmos que apenas 3,3\% das mulheres ativas relataram níveis moderados de sintomas climatéricos.

Taivora \& De Lorenzi ${ }^{12}$ verificaram que os níveis de sintomas climatéricos mostraram-se significativamente menos severos no grupo de mulheres mais ativas. Em seus estudos, $63,6 \%$ das mulheres sedentárias relataram sintomas climatéricos de intensidade moderada a severa, contra $33,4 \%$ das fisicamente ativas.

Por meio da análise de regressão linear, ficou evidenciado em nosso estudo, que se a mulher é ativa fisicamente, o IMBK tende a diminuir em 12 pontos. Esse resultado significativo reforça o fato de que a atividade física regular pode amenizar a intensidade dos sintomas climatéricos.

Observou-se neste estudo que dentre todos os sintomas avaliados, os de intensidades mais severas foram os referentes às ondas de calor ou fogachos, seguido por insônia, os quais embora aparecendo em níveis leves nas mulheres ativas mostraram-se bem mais intensos (nível severo) nas insuficientemente ativas. Ainda que o mecanismo envolvido no alívio das queixas vasomotoras não seja totalmente conhecido, algumas evidências sugerem que o exercício físico regular e/ou mais vigoroso promo- 
va um aumento das $\beta$-endorfinas hipotalâmicas, estabilizando a termorregulação hipotalâmica ${ }^{11}$. Em um estudo realizado na Suécia, somente 5\% das mulheres com atividade física regular apresentaram fogachos intensos, em comparação a um número entre $14 \%$ e $16 \%$ das mulheres que se exercitavam pouco ou nada por semana ${ }^{19}$.

Outro estudo realizado, em uma linha associada mostrou uma tendência de que a maioria das mulheres climatéricas pode se revelar insatisfeitas com o tempo e qualidade do sono. Esta condição que em relação às demais queixas climatéricas que tendem a melhorar com o passar do tempo, parecem, ao contrário, se intensificar nos anos que se seguem a menopausa ${ }^{20}$. Contudo, ainda não foi possível estabelecer uma relação definitiva entre os distúrbios do sono e as oscilações hormonais que caracterizam essa fase, embora algumas evidências tenham sugerido que tal desiquilíbrio possa estar relacionado à severidade dos sintomas vasomotores ou a transtornos psíquicos ${ }^{21}$.

Conforme tem sido defendido por alguns autores, existem probabilidades de que mulheres em transição para menopausa ou pós-menopausadas possam apresentar problemas de sono com uma frequência muito maior do que mulheres mais jovens em pré-menopausa ${ }^{22}$. Entre as prováveis causas de insônia ou transtornos de sono associados à menopausa, destacam-se a ocorrência de sintomas vasomotores (por exemplo, fogachos, suores noturnos), quadros depressivos e transtornos respiratórios durante o sono (como apneia do sono), além de quadros de dor crônica ${ }^{23,24}$.

Alguns estudos afirmam que persistem controvérsias sobre o quanto tais queixas são decorrentes somente da queda estrogênica ou de fatores psicossociais relacionados ao próprio processo de envelhecimento. As ondas de calor e a atrofia urogenital seriam as únicas queixas diretamente relacionadas à queda dos níveis estrogênicos. As demais estariam relacionadas principalmente à dificuldade de aceitar o processo de envelhecimento, este simbolizado pela menopausa ${ }^{25,26}$.

Ainda sem sustentação de base científica sólida, algumas sugestões procedem na explicação de que os sintomas climatéricos parecem ser menos intensos em mulheres com maior nível educacional, maior renda familiar e nas que se exercitam regularmente ${ }^{18}$. O efeito do nível educacional é pensado em função de que uma maior escolaridade não apenas facilita o acesso à informação sobre a natureza do processo do climatério, como também, em consequência, pode reduzir a ansiedade comum nessa fase ${ }^{7}$. Verificou-se, também, neste presente estudo que $36,7 \%$ das mulheres ativas possuíam curso superior completo e $23,3 \%$ pós-graduação. Em contrapartida no grupo das mulheres insuficientemente ativas, apenas $23,3 \%$ e $6,7 \%$ possuíam curso superior completo e pós-graduação, respectivamente. Estes resultados embora não tendo revelado estatística significativa entre os grupos, ajudam como informações adicionais relevantes que podem servir de referência para estudos mais detalhados e específicos para a identificação de possíveis relações entre atividade física e o climatério.

Para mulher climatérica, a prática de atividade física regular, ainda que de pouca intensidade, parece contribuir globalmente para uma melhoria das condições de saúde, tendo um impacto positivo também na sua esfera psíquica e em muitos dos sintomas característicos dessa fase, melhorando, inclusive, seus sentimentos e atitudes ${ }^{27}$.

A sintomatologia climatérica apresentou-se menos intensa no grupo de mulheres ativas fisicamente, quando comparadas às insuficientemente ativas. A maioria das mulheres ativas foi classificada como portadoras de sintomas leves do climatério (o melhor nível da escala), enquanto as insuficientemente ativas obtiveram prevalência de sintoma de intensidade moderada. Nesse sentido, o presente estudo, demonstrou que a prática de atividades físicas regulares pode contribuir po- 
sitivamente para a redução dos sintomas climatéricos de mulheres de meia idade. Ainda que, embora apresentando alguns indicativos de diminuição em alguns sintomas do climatério, tipos de atividades físicas como as descritas para o grupo de atividades físicas insuficientes, não atendem a uma minimização efetiva dos eventos negativos que o climatério provoca nestas mulheres. Esta última afirmativa, não acompanha os resultados de alguns estudos apresentados acima, visto que os mesmos relatam benefícios da atividade física regular e de baixa intensidade para a saúde da mulher. Talvez aqueles resultados possam ter referência com índices de atividade física determinado por outro instrumento que não o aqui utilizado.

Considerando-se o fato de que na definição do índice do grupo de mulheres insuficientemente ativas, obedeceu-se o ponto de corte determinado pelo IPAQ e que a média das atividades destas ficou bem próxima ao extremo do ponto de corte para tal índice (129 minutos semanais), poder-se-ia dizer aqui que as mesmas se enquadram como sendo um grupo de atividade física regular. Convém se enfatizar aqui que embora seja, o IPAQ, um instrumento proposto pelo Grupo Internacional para Consenso em Medidas da Atividade Física (constituído sob a chancela da Organização Mundial da Saúde) e amplamente utilizado em pesquisas associadas ao estado físico da mulher ${ }^{28}$, há de se admitir, a bem da fidedignidade dos resultados aqui chegados, uma possibilidade de limitação do instrumento que foi utilizado na definição dos grupos. A falta de um diagnóstico clínico específico para avaliar as condições orgânicas das participantes deste estudo e/ou uma verificação mais direta do índice físico delas poderiam ser relevantes em termos de se definir, mais claramente, a relação entre atividade física e os problemas do climatério estudados.

Portanto, a despeito dos achados indicando que diferentemente do nível de atividade física das mulheres ativas, o das mulheres insuficientemente ativas não se mostrar suficiente para minimizar os efeitos do climatério nelas, estes resultados devem ser interpretados à luz de suas limitações. Assim sendo, desenhos de pesquisa alternativos, tais quais os vinculados a modelos longitudinais e com instrumentação para avaliações físicas mais específicas poderão vir a oferecer novos conhecimentos sobre os processos psicobiológicos relacionados à sexualidade de mulheres na menopausa.

\section{Contribuiç̧ão dos autores}

Probo AMP e Cabral PUL contribuíram com a concepção do projeto, procedimentos referentes a seleção da amostra e coleta de dados, análise e interpretação dos dados. Probo AMP; Soares NIS; Silva VF e Cabral PUL colaboraram com a redação do artigo e revisão crítica relevante do conteúdo intelectual. Soares NIS; Silva VF e Cabral PUL cooperaram com a aprovação final da versão a ser publicada.

\section{Referências}

1. Spritzer PM, Wender MCO. Terapia hormonal na menopausa: quando não usar. Arq Bras Endocrinol Metab. 2007;51(7):1058-63.

2. Wu X, Cai H, Kallianpur A, Gao YT, Yang G, Chow WH, et al. Age at menarche and natural menopause and number of reproductive years in association with mortality: results from a median follow-up of 11.2 years among 31,955 naturally menopausal Chinese women. PLoS One. 2014;9(8):e103673.

3. De Lorenzi DRS, Danelon C, Saciloto B, Padilha Junior I. Fatores indicadores da sintomatologia climatérica. Rev Bras Ginecol Obstet. 2005;27(1):7-11.

4. Bulcão CB, Carange E, Carvalho HP, Ferreira-França JB, Kligerman-Antunes J, Backkes J, et al. Aspectos fisiológicos, cognitivos e psicossociais da senescência sexual. Cien Cogn. 2004;1(1):54-75.

5. De Lorenzi DRS. Avaliação da qualidade de vida no climatério. Rev Bras Ginecol Obstet. 2008;30(3):103-6. 
6. Cheng MH, Lee SJ, Wang PH, Fuh JL, Wang SJ. Does menopausal transition affect the quality of life? A longitudinal study of middle-aged women in Kinmen. Menopause. 2007;14(5):885-890.

7. De Lorenzi DRS, Catan LB, Moreira K, Ártico GR. Assistência à mulher climatérica: novos paradigmas. Rev bras enferm. 2009;62(2):287-93.

8. Skrzypulec V, Dabrowska J, Drosdzol A. The influence of physical activity level on climacteric symptoms in menopausal women. Climacteric. 2010;13(4):355-61.

9. Kim MJ, Cho J, Ahn Y, Yim G, Park HY. Association between physical activity and menopausal symptoms in perimenopausal women. BMC Womens Health. 2014;14:122.

10.Zanesco A, Zaros PR. Exercício físico e menopausa. Rev Bras Ginecol Obstet. 2009;31(5):254-61.

11. Elavsky S. Physical activity, menopause and quality of life: The role of affect and selfworth across time. Menopause. 2009;16(2):265-271.

12. Tairova OS, De Lorenzi DRS. Influência do exercício físico na qualidade de vida de mulheres na pós-menopausa: um estudo caso-controle. Rev Bras Geriatr Gerontol. 2011;14(1):135-45.

13. De Azevedo Guimarães AC, Baptista F. Influence of habitual physical activity on the symptoms of climacterium/menopause and the quality of life of middle-aged women. Int J Womens Health. 2011;3:319-328.

14. Borkoles E, Reynolds N, Ski CF, Stojanovska L, Thompson DR, Polman RC. Relationship between Type-D Personality, Physical Activity Behaviour and Climacteric Symptoms. BMC Women's Health. 2015;15:18.

15. Barreto HVA, Alves TTM, Soares NIS, Silva VF, Cabral PUL. Atividade física na saúde e qualidade de vida de mulheres climatéricas. Cinergis. 2015;16(3):203-208.

16. Kupperman HS, Blatt MH, Wiesbader H, Filler W. Comparative clinical evaluation of estrogenic preparations by the menopausal and amenorrheal indices. J Clin Endocrinol Metab. 1953;13(6):688-703.

17. Matsudo SM, Araujo T, Matsudo VR, Andrade D, Andrade E, Oliveira LC, et al. International Physical Activity Questionnaire (IPAQ): study of validity and reliability in Brazil. Rev Bras Ativ Fís Saúde. 2001;6(2):5-18.

18. Canário AC, Cabral PU, Spyrides MH, Giraldo PC, Eleutério J Jr, Gonçalves AK. The impact of physical activity on menopausal symptoms in middle-aged women. Int J Gynaecol Obstet. 2012;118(1):34-6.

19. Ivarson T, Spertz AC, Hammar M. Physical exercise and vasomotor symptoms in postmenopausal women. Maturitas. 1998;29(2):139-46.

20. De Lorenzi DRS, Baracat EC, Saciloto B, Padilha Júnior I. Fatores associados à qualidade de vida após menopausa. Rev Assoc Med Bras. 2006;52(5):312-7.

21. Souza CL, Aldrighi JM. Sono e Climatério. Reprod Clim. 2001;16(1):20-25.

22. Dennerstein L, Lehert P, Guthrie J. The effects of the menopausal transition and biopsychosocial factors on well-being. Arch Women Ment Health. 2002;5:15-22.

23. Joffe H, Soares CN, Cohen LS. Assessment and treatment of hot flushes and menopausal mood disturbance. Psychiatr Clin North Am. 2003;26:563-580.

24. Moline M, Broch L, Zak R. Sleep problems across the life cycle in women. Curr Treat Options Neurol. 2004;6(4):319-330.

25. De Lorenzi DRS, Saciloto B. Frequência da atividade sexual em mulheres menopausadas. Rev Assoc Med Bras. 2006;52(4):256-60.

26. Lind-Åstrand L, Hoffmann M, Hammar M, Kjellgren KI. Women's conception of the menopausal transition - a qualitative study. J Clin Nurs. 2007;16(3):509-17.

27. Kakkar V, Kaur D, Chopra K, Kaur A, Kaur IP. Assessment of the variation in menopausal symptoms with age, education and working/non-working status in north-Indian sub population using menopause rating scale (MRS). Maturitas. 2007;57(3):306-14.

28. Craig CL, Marshall AL, Sjostrom M, Bauman AE, Booth ML, Ainsworth BE, et al. International physical activity questionnaire: 12 -country reliability and validity. Med Sci Sports Exerc. 2003;35:1381-95.

ENDEREÇO PARA CORRESPONDÊNCIA NATHANAEL IBSEN DA SILVA SOARES naelibsen@hotmail.com.
Rua Carlos Alberto de Moraes, $\mathrm{n}^{\circ} 519$

Cep: 65633-150, Parque Alvorada,

Timon - MA Telefone: (86) 9829- 0853 